\title{
Development of discrimination technology associated to protection secondary circuits in Smart Substation Based on Semantic Features of SCD
}

\author{
Wei $\mathrm{BAO}^{1}$, Xiang $\mathrm{GAO}^{2}$, Ke $\mathrm{WANG}^{3}$, Qi Geng ${ }^{3}$, Jie $\mathrm{SONG}^{1}$, Ping ZENG ${ }^{1}$, Desheng $\mathrm{ZHOU}^{1}$ \\ ${ }^{1}$ State Grid Shanghai Electric Power Company, Shanghai, China \\ ${ }^{2}$ Shanghai Yihao Automation Co., Ltd, Shanghai, China \\ ${ }^{3}$ School of Electronic Information and Electrical Engineering, Shanghai Jiao Tong University, Shanghai, China
}

\begin{abstract}
As a core part of smart substation, SCD files make secondary circuits in smart substation becoming a 'black box', which brings great difficulties to operation and maintenance. This paper proposes the SCD virtual circuit identification technology based on a semantic strength, which can provide an effective technical solution for the SCD management of smart substation, and demonstrates a reference for controlling the maintenance risk of smart substation.
\end{abstract}

\section{Introduction}

Since the strategic promotion of SGCC's smart grid in 2009, a large number of smart substations have entered the engineering application. China has become the country with the most smart substations [1-3]. The smart substation completes the configuration of the secondary system signal correlation based on the substation configuration file SCD. Since the SCD is based on the XML format, the performance characteristic of the secondary system is greatly different from that of the conventional integrated system which are characterized by hard wiring cables. The invisibility brings great difficulties for the operation and maintenance of field workings.

In order to standardize the secondary system design and engineering application of smart substation, State Grid Corporation has promulgated Q/GDW 1161-2014 Standardization Design Specification for Transmission Line Protection and Auxiliary Equipment and Q/GDW 1175-2013 Standardization Design Specification for Power Transformer, High Voltage Shunt Reactor, Bus Bar Protection and Auxiliary Equipment. Therefore, it provides the basis for the design and engineering application of the secondary system in smart substation.

In this paper, the semantic-based SCD virtual loop identification technology is proposed. Through hierarchical analysis of SCD files, the relationship between virtual circuits in smart substation is determined, which provides reliable technical support for smart substation engineering application. At the same time, it is clear that the virtual loop semantic consists of tag semantics and structure semantics. Label semantics, is to identify virtual circuits. Structural semantics, is to identify the functional integrity and the correctness of virtual circuits. However, any semantics, especially tag semantics, have different strength, which are constrained by different levels of standards and norms.

\section{SCD virtual circuit application analysis}

\subsection{Describing characteristics of SCD virtual circuit}

The description of the virtual circuit in the SCD file is indirect and implicit, with no explicit description of the objectification. For example, sending a virtual circuit of a remote trip command, the description of the external part of protection in SCD is as follows:

<ExtRef intAddr="PI/GOINGGIO13.SPCSO1.stVal" doName $=" T r " \quad$ lnInst="5" iedName="PM2201B" daName="general" ldInst="PI" lnClass="PTRC" prefix $="$ ">

This circuit is the one that sends the remote trip command to PL2201B by the busbar protection PM2201B. The object description should contain three parts:

(1) Circuit name.

(2) Virtual sending terminal.

(3) Virtual receiving terminal.

Therefore, the objectification of virtual circuits is an important basis for achieving identification conforming to the principles of protection design.

The legitimacy of FCDA is through the fact that LD, $\mathrm{LN}$, DO, and DA in the FCDA can be found in the corresponding LD/LN/DO/DA path of the IED.

The correspondence between ExtRef and FCDA is: Can $\mathrm{LD}+\mathrm{LN}+\mathrm{DO}+\mathrm{DA}$ in each ExtRef be found the 
corresponding $\mathrm{LD}+\mathrm{LN}+\mathrm{DO}+\mathrm{DA}$ in the FCDA of its specified iedName.

If you meet:

(1) FCDA legal.

(2) ExtRef is able to be found the corresponding FCDA.

Then the virtual circuit can be established. The next step is to check ExtRef to determine whether the meaning of the input circuit meets the requirements of the 1161 and 1175 specifications. For the output circuit in the specification, this can be done by checking FCDA

\subsection{Visualization based on work characteristics}

Because SCD is only the function carrier of the secondary system, the object of work management should be a functional secondary circuit. Therefore, the SCD-based implementation of the smart substation does not change the substation service features, but the substation secondary system appears as a 'black box'. Therefore, visualization based on business characteristics of the SCD virtual circuit is a basic application need, shown as Figure 3.

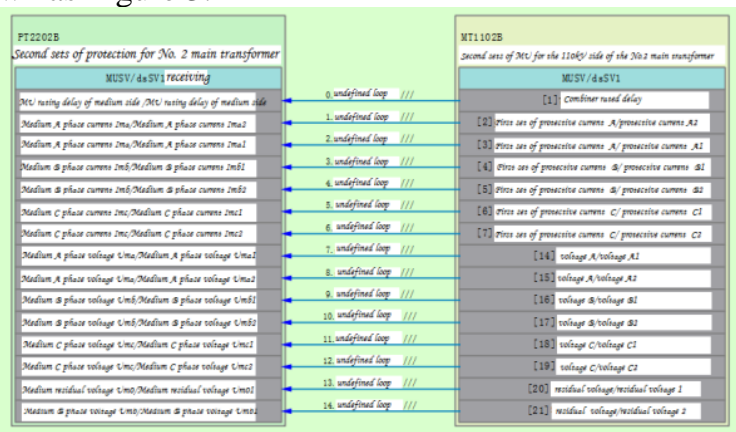

Fig.3 Visualization of virtual circuits

However, there is a certain difference in the description of secondary virtual circuits in SCD given by different IED produced by varying manufacturers.

Specifically: Beijing Sifang company 500kV CSC101 line protection used to start the remote trip order with GOOSE virtual terminal address: PI/GOINGGIO13.SPCS01.stVal, and its description is 'other protection stop'. NARI PCS931-GM dual configured protection virtual terminal address is PIGO/GOINGGIO5. SPCS01.stVal, the corresponding description is 'send remote trip'.

\subsection{Problems found in engineering application}

In practical engineering applications, many problems in SCD virtual circuits are found, such as:

(1) Start-Breaker-Failure circuit missing

A line bay in a $220 \mathrm{kV}$ smart substation SCD file lacks a Start-Breaker-Failure circuit, shown as Figure 4.

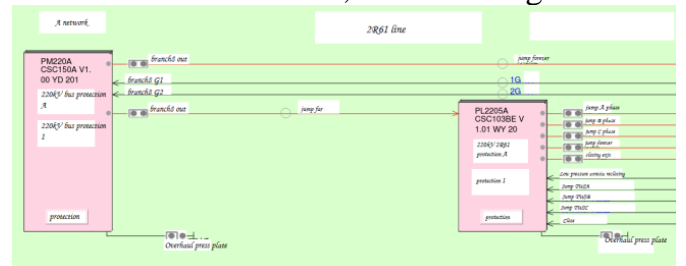

Fig.4 Lack of Start-Breaker-Failure circuit
(2) Misconnection of the secondary circuits

Short circuit protection in a substation SCD file has the phenomenon of secondary virtual circuit error, as shown in Figure 5

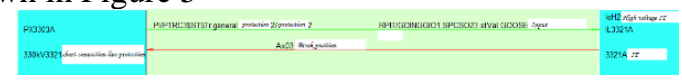

Fig.5 Wrong connection in a secondary circuit

This circuit is described in the SCD file as follows:

$<$ ExtRef daName $=$ " $\mathrm{t}$ " doName $=$ "Pos" iedName $=$ "IL3321A" intAddr = "PI / GOINGGIO1.DPCSO1.stVal" ldInst = "RPIT" lnClass = "XSWI" lnInst = "1" prefix = "QG3" />

The reason is that the output terminal of the secondary breaker position circuit is incorrectly configured to the breaker displacement time ' $t$ '.

(3) Circuits of non-operative secondary equipment is not complete

As the construction of substations is generally carried out in stages, during a certain time when the protection equipment has been in motion in some operational bays of smart substation, but the SCD file has not configured the corresponding virtual circuit timely, the second virtual circuit of the protection is completely missing, shown as Figure 6.

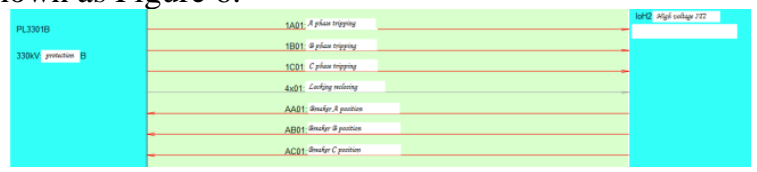

Figure 6 No configuration in a secondary circuit

Therefore, exploring the identification technique of virtual circuits in SCD has become the key to ensure the reliable operation of smart substations.

\section{SCD identification scheme analysis}

\subsection{Template library based on common design rules}

The 'Substation General Design Specification' issued by State Grid Corporation defines the secondary system design specifications for substations, which stipulate the principle of secondary system configuration. Combined with a typical substation wiring mode, it is possible to generally determine the secondary virtual circuit expressed in the SCD, and constitute the rules of the second system identification [9].

On this basis, the identification needs to be combined with the networking scheme of substation process levels. The contents of the identification include:

(1) The functional circuit connection between IEDs, which verifies whether the basic functional circuit is complete and conforms to the secondary system design rules. This verification is essentially a structural semantic verification, the core is complete and consistent

(2) The normalization and correctness of functional circuit network communication parameters, which verifies whether there is a network parameter error. This verification is essentially a tag semantic verification, the core is correctness and equivalence.

For example, (1) in 1.3 belongs to the semantic tag error. (2) in 1.3 belongs to the structure semantic error. 
There are differences between the two error bearers: the object of tag semantic error is a circuit object, and the object of structure semantic errors is a function, or a functional protective device, or a substation that contains a protective device.

\subsection{Function-based circuit identification}

Logical Node (LN) defined by IEC61850 is the carrier and manifestation of function implementation. It displays and controls the inputs, outputs, settings and parameters related to the function implementation. That is, the LN can make all the inputs and settings of 'Function' both visible and configurable, and also make all the outputs of "Function" orientable and verifiable. Therefore, the circuit identification for secondary system functions can be performed step-by-step along the path from voltage class, within-class bay, within-bay device, and within-device functions. Such as:

(1) The integrity of the functional circuit logic closed loop: Take line protection as an example, the complete logic closed loop includes analog and digital sampling, protection trip action output and other circuits.

(2)The correctness of the virtual circuit connection: According to the type and description of the secondary virtual circuit configured in the SCD file, the type characteristics of the circuit are extracted, matched and compared with the rule base, and the integrity of the circuit connection is checked ${ }^{[10]}$.

\subsection{Identification based on communication description mechanism}

For the transmission of information between IED, IEC 61850 standard generally achieves through the GOOSE and SMV data description. Therefore, the identification of SCD virtual circuits needs to be combined with reporting control block features in the SCD, data set definitions, communication parameters and other elements. The contents of identification includes:

(1) The communication address configuration format and scope of GOOSE and SMV report control blocks is in line with the norms.

(2) APPID, MAC address is repeated or missing.

(3) IP address assignment is reasonable, whether there is duplication or missing.

Integrators of smart substations modify the SCD at the construction site, and generally configure their secondary circuit connections manually through their dedicated SCD configuration tools. Although some integrators' tools have template-based configuration aids, the diversity of combinations between protection and smart devices still poses a significant challenge to the correctness of the SCD engineering configuration ${ }^{[6-8]}$.

\section{Virtual circuit identification technology based on semantic strength}

\author{
4.1 Virtual circuit identification scheme based \\ on semantic strength
}

Given the strength and weakness of SCD semantics, the layer-by-layer identification of the SCD virtual circuit can be implemented according to the semantic strength. Specifically, based on the requirements of IEC61850-6, the grammatical structure of the secondary virtual circuit in the SCD input is formally identified. Based on the Technical Specifications for Smart Substation Configuration Files issued by State Grid Corporation of China, the relationship between the IED device and primary system is identified. IED device model description normative identification is conducted based on1396 standard. Based on 1161, 1175 design specifications, the reference path and DESC the identification of secondary virtual circuit is conducted, as shown in Figure 1.

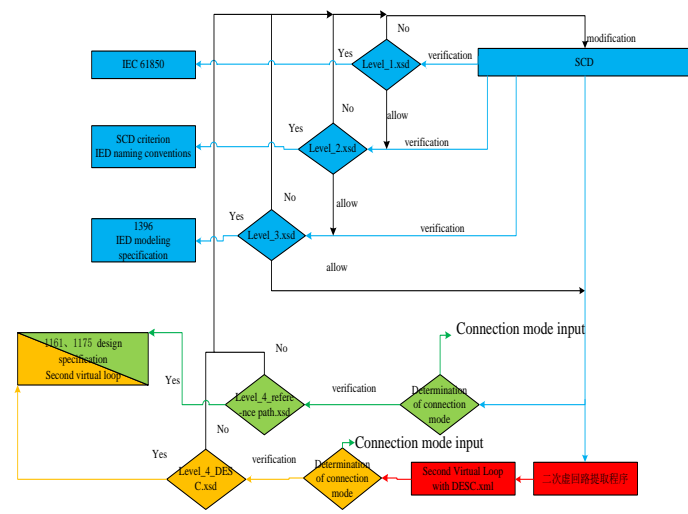

Fig.1 Virtual circuit identification diagram based on semantic strength

\subsection{Implementation analysis Based on XML Schema}

XML Schema is a language that creates schema definitions in XML, with the file extension xsd. The Schema document itself is written in XML language and supports multiple programming forms such as include and import. Schema documents specify the XML file so that its correctness definition structure is clear and defined. The Schema document gives the syntax and semantics of the specified XML file. The identification is performed by a specific program or software, such as the xml.validation package in a language such as JAVA, software such as XMLSpy, Oxygen.

The Schema pattern language can not only specify the grammar structure of the XML document, but also achieve a certain degree of semantic inspection through consistency constraints. There are two kinds of consistency constraints used in Schema pattern language:

(1) Uniqueness constraint, that is, xs: unique, whose role is to specify the uniqueness of a certain element's value or attribute within a certain range.

(2) The key constraints, xs: key and xs: keyref, are functions that use a set of values or attributes of the keyref that must refer to the set of values or attributes of their corresponding key.

The substation connection, secondary circuits between IED and other information is expressed through a data set of FCDA expression output and ExtRef expression input to complete, Schema language model can only describe the value of a single element or 
attribute constraints, it cannot be used directly to describe the correctness of the connection or indirect information such as secondary circuit definition. Therefore, it is impossible to conduct normative identification of SCD virtual circuits by merely using Schema document.

The description mechanism of secondary circuits in SCD determines that it cannot be directly identified by Schema language constraints. But SCD itself does not lack any configuration information in the substation, instead a lot of information coupled together makes it difficult to deal with. Therefore, the information in the SCD file needs to be extracted according to the type, that is, the decoupling of different types of information, thereby achieving the integration of the similar information in the SCD file that is actually in close contact with each other. After the form of the document constraint is written to a new XML document, the inspection rules are described in a Schema document for SCD virtual circuit identification.

\subsection{Design specifications of smart substation}

It can be seen from the above, IEC61850, 1396 specification, 1161 specification and 1175 specification, are the main levels of identification framework. However, from the perspective of virtual circuits, the semantic strength of their constraints focus on different points:

(1) IEC61850 focuses more on tag semantics, even for the constraints of the structure, IEC 61850 is more for communication purposes, not the circuit structure. It should be used as the basis for the label check of virtual circuit identification;

(2) 1396 specification belongs to the category of structural semantics, and refines part of the label semantics, but the structure focuses on the general structure of the substation. It should therefore be used as a circuit check to reinforce the semantic of the tag and the semantic basis of the infrastructure;

(3) 1161 and 1175 specifications, the device type as the object, detailed provisions of the device input and output, and model expression constraints, belong to the strong structural semantics. It should be used as a circuit check to strengthen the structural semantic basis.

The Schema model provided by IEC61850, while only expressing the constraint of IEC61850, is essentially a formal expression of syntactic that can be used as an input for automatic checking. Therefore, regardless of 1396 , or 1161,1175 involved in the syntactical constraints, can be used to expand the Schema way, then the strength of automatic inspection also will be strengthened.

In addition, the 1161 and 1175 specifications of circuit design have more realistic value. In this paper, the circuit belongs to the highest intensity semantic basis. Although the standard form of expression relies mainly on tables and words, its essence is thesaurus, word bag, word vector in semantics, as an examination of the equivalence relation, the current technology is already sufficient. With reference to the current development of AI technology and mathematics, a complete computing system of 'greater than', 'less than', 'addition', and "subtraction" is extended from the single operation of 'equal to' Semantic 'distance', so that the 'strength', 'complete' and other properties corresponding to the circuit semantic checks will have more quantitative features.

\section{Typical project case analysis}

In order to verify the effectiveness of the technical solution, a $220 \mathrm{kV}$ smart substation SCD was selected and the semantic-based Schema technique was used to do the layer-by-layer normative identification of the virtual circuit for the selected SCD as follows:

The first step: IEC 61850 inspection, XML-based original SCD file and IEC61850-6 given XSD file consistency comparison, the main goal is to check whether SCD syntax structure is normative, the test results are as follows:

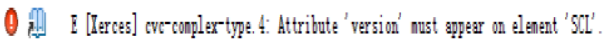

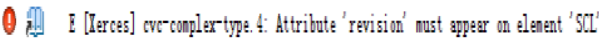

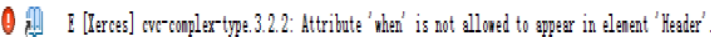

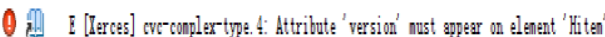

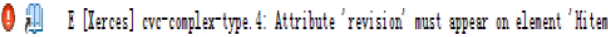

The first three example differences are:

(a) The required attribute "version" is missing from the element "SCL";

(a) The required attribute "revision" is missing from the element "SCL";

(c) The element "Header" does not allow the attribute "when".

The lack of these attributes mainly affects the validity of the SCD version management identity.

The second step is to check the consistency of the original SCD file and the XSD file, based on the inspection of the SCD technical specification through XML Schema technology. Check if the SCD meets the SCD specification, as follows:

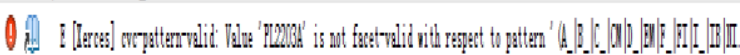

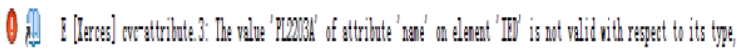

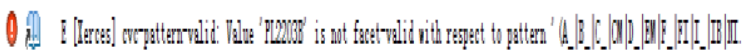

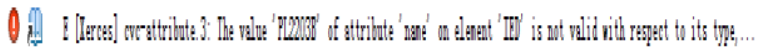

The two issues reflected by the above four differences are:

(a) 'PL2203A' does not conform to the IED naming scheme for SCD specification appendix $\mathrm{J}$.

(b) 'PL2203B' does not conform to the IED naming scheme for SCD specification appendix J.

Non-standard naming will affect the correct judgment of the nature of the protection.

The third step IED configuration check, based on Q/GDW 1396-2012 Data Model of Protection Relay in Project Based on IEC61850, was conducted by the comparison of SCD files and XSD file, as follows: 


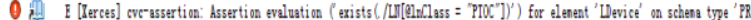

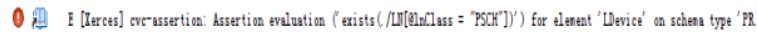

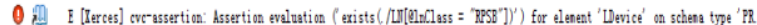

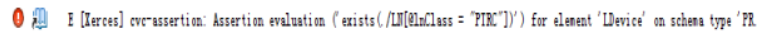

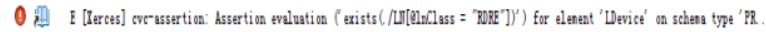

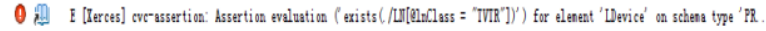

Inspection results reflect the six differences are:

(a) The required logical node named PTOC is missing from the PROT LDevice of 'PL2203A'.

(b) The required logical node named $\mathrm{PSCH}$ is missing from the PROT LDevice of 'PL2203A'.

(c) The required logical node named RPSB is missing from the PROT LDevice of 'PL2203A'.

(d) The required logical node named PTRC is missing from the PROT LDevice of 'PL2203A'.

(e) The required logical node named RDRE is missing from the PROT LDevice of 'PL2203A'.

(f) The required logical node named TVTR is missing from the PROT LDevice of 'PL2203A'.

Without these logic nodes, the Q/GDW 1161-2014 Standardization Design Specification for Transmission Line Protection and Auxiliary Equipment cannot be invoked directly when checking the virtual circuit associated with this IED. Also the Q/GDW 1175-2013 Standardization Design Specification for Power Transformer, High Voltage Shunt Reactor, Bus Bar Protection and Auxiliary Equipment.

The fourth step is mainly based on $Q / G D W$ 1161-2014 Standardization Design Specification for Transmission Line Protection and Auxiliary Equipment, and $Q / G D W \quad 1175-2013$ Standardization Design Specification for Power Transformer, High Voltage Shunt Reactor, Bus Bar Protection and Auxiliary Equipment. The check is conducted through two methods:

(1) Based on the reference path inspection.

(2) Based on the description of DESC information of DO.

Considering that the effective output circuit of each device is reflected in the input of the opposite end, it is necessary to extract the local terminal dodesc information and the opposite terminal dodesc information of each IED input virtual circuit in the SCD so as to form a secondary virtual circuit system integrity check. Specific results are as follows:

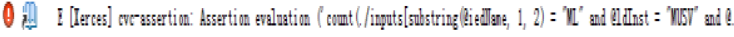

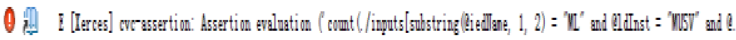

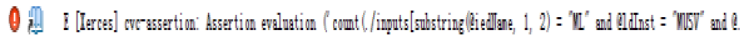

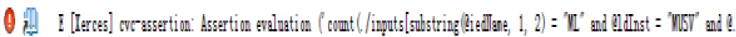

Example of result, 4 differences are:

(a) There is only one synchronous voltage in the input virtual circuit of 'PL2203B', which is different from the two synchronous voltage inputs in the standard.

(b) There is only one synchronous voltage in the input virtual circuit of 'PL2204B', which is different from the two synchronous voltage inputs in the standard.

(c) There is only one synchronous voltage in the input virtual circuit of 'PL2205B', which is different from the two synchronous voltage inputs in the standard.

(d) There is only one synchronous voltage in the input virtual circuit of 'PL2206B', which is different from the two synchronous voltage inputs in the standard.

The four results above reveal that line protection does not use double $\mathrm{A} / \mathrm{D}$ synchronous voltage application mode.

\section{Conclusion}

In this paper, SCD virtual circuit identification technology based on semantic strength is proposed for the application of SCD in smart substations. The validity of this scheme is verified by checking SCD files in engineering application. Ensuring the correctness of SCD files is the basis for realizing SCD's life-cycle management in smart substations[11], and it is the prerequisite for the implementation of the national regulation No. 80, the on-line monitoring and intelligent diagnosis of the protection system, and the construction of the secondary safety early-warning system for smart substations ${ }^{[12]}$. The solution proposed in this paper is of great value to the operation safety of smart substation protection system and provides a reliable technical guarantee for the promotion of engineering practice of smart substations.

\section{References}

1. HUANG Yizhuang. Smart substation is the further objective of SAS[J]. Power System Protection and Control, 2013, 41, 2, pp. 88-92.

2. BAO Wei, GAO Xiang, SHEN Bing, ZHOU Jian. Research method of "non-intruding test" for smart substation[J]. Power System Protection and Control, 2015, 43, 20, pp.125-129.

3. GAO Zhiyuan, YAO Jianguo, GUO Kunya, et al Study on the supporting role of smart grid to the construction of smart city[J]. Power System Protection and Control, 2015, 43, 11, pp.148-153.

4. GAO Xiang, YANG Yijun, JIANG Jianning, WU Zhenjie. Introduction and analysis of main technical scheme of two loop monitoring based on SCD[J]. Power System Protection and Control, 2014, 42, 15, pp.149-154.

5. HU Daoxu, WO Jiandong, et al. Virtual loop system of intelligent substation based on IEC61850[J]. Automation of Electric Power System, 2014, 34, 7, pp.78-82.

6. YI Yang, HU Fangkai, ZHOU Yu, et al. Research on verification method of SCD file two-dimensional code in Intelligent substation[J]. Power System Protection and Control, 2015, 43, 2, pp.113-118.

7. WANG Song, XUAN Xiaohua, LU Chengyu. Intelligent substation configuration file version management method[J]. Automation of Electric Power Systems, 2013, 37, 17, pp.95-98.

8. ZHENG Wenbing, WANG Ruibiao, et al. Privilege management for SCD version of intelligent substation based on virtual terminal modification[J]. Smart Grid, 2015, 3, 9, pp.792-796.

9. WU Hengfu, DOU Huiguang, XIANG Qian, et al. Specification checking of SCD files in intelligent substation based on design specification[J]. Power 
System and Clean Energy, 2015, 31, 4, pp.84-88.

10. BAO Wei, GAO Xiang, TU Qi, et al. The development of SCD management method based on correct checking[J]. Electrical Engineering, 2016, 6, pp.133-137.

11. ZHANG Perchao, JIANG Jianning, YANG Yijun, LIU Shaojun. Lifecycle management of configuration information for Smart Substation[J]. Automation of Electric Power Systems, 2014, 38 ,10, pp.85-89.

12. XU Changbao, ZHANG Chen, JIANG Hongtu. Technical research of secondary equipment's state monitoring in smart substation[J]. Power System Protection and Control, 2015, 43, 7, pp.127-131. 